\title{
Atypical Traumatic Pneumorrhachis Accompanied by Paraparesis
}

\author{
Kweon Young Kim, MD, Jung Hun Kang, MD, Min Hong Lee, MD, Yong Han, MD, Dae Woo Choi, MD
}

Department of Rehabilitation Medicine, Chosun University Hospital, Gwangju, Korea

Pneumorrhachis, caused by intraspinal air, is an exceptional but important radiographic finding that is accompanied by different etiologies. Pneumorrhachis, by itself, is usually asymptomatic and gets reabsorbed spontaneously. Therefore, the patients with pneumorrhachis are mostly managed conservatively. We encountered a unique case of atypical traumatic pneumorrhachis accompanied by paraparesis.

Keywords Pneumorrhachis, Intraspinal air, Paraparesis

\section{INTRODUCTION}

Pneumorrhachis (PR), which is characterized by air formed within the spinal canal, is a very rare condition that is generally associated with trauma and surgical procedures. The causes can be classified as iatrogenic, nontraumatic and traumatic, which is the rarest among the three. The precise mechanisms are unknown. PR is usually asymptomatic and clinically non-specific, and it does not tend to migrate, but it gets reabsorbed spontaneously into the bloodstream over the period of several days. Therefore, the patients with PR are normally managed by conservatively treatment $[1,2]$.

Although PR arising secondarily to traumatic causes is rare, it has been observed in the patients with bone frac-

Received June 10, 2013; Accepted August 23, 2013

Corresponding author: Dae Woo Choi

Department of Rehabilitation Medicine, Chosun University Hospital, 365 Pilmun-daero, Dong-gu, Gwangju 501-717, Korea

Tel: +82-62-220-3153, Fax: +82-62-223-0245, E-mail: dwchoi81@naver. com

(c) This is an open-access article distributed under the terms of the Creative Commons Attribution Non-Commercial License (http://creativecommons. org/licenses/by-nc/3.0) which permits unrestricted noncommercial use, distribution, and reproduction in any medium, provided the original work is properly cited.

Copyright $\odot 2014$ by Korean Academy of Rehabilitation Medicine tures and spinal injuries. Presenting with pain or neurological injuries are uncommon, thus they are usually diagnosed accidentally by radiographic imaging, such as computed tomography (CT) $[1,2]$. This paper reports a rare case of an atypical traumatic pneumorrhachis accompanied by paraparesis; reviews of literature are also included for references.

\section{CASE REPORT}

A 51-year-old male was admitted to the hospital after a chest trauma caused by ironware which fell from the tractor. He presented with chest pain and difficulty in breathing.

Upon the arrival, his vital signs were normal, and the chest radiograph revealed fractures of the right 2 nd to 4th and left 1st to 6 th ribs, accompanied by a hemopneumothorax. A closed reduction and lung suture were performed immediately. CT revealed a diffuse air in thoracic epidural space, but there were no clinical symptoms. Therefore conservative treatment was conducted (Fig. 1).

After the surgical procedures, the patient complained of dysuria, dyschezia, and motor weakness and numbness in the both lower limbs. On the physical examination, the muscle tone of the upper limbs were normal, hypertonia 

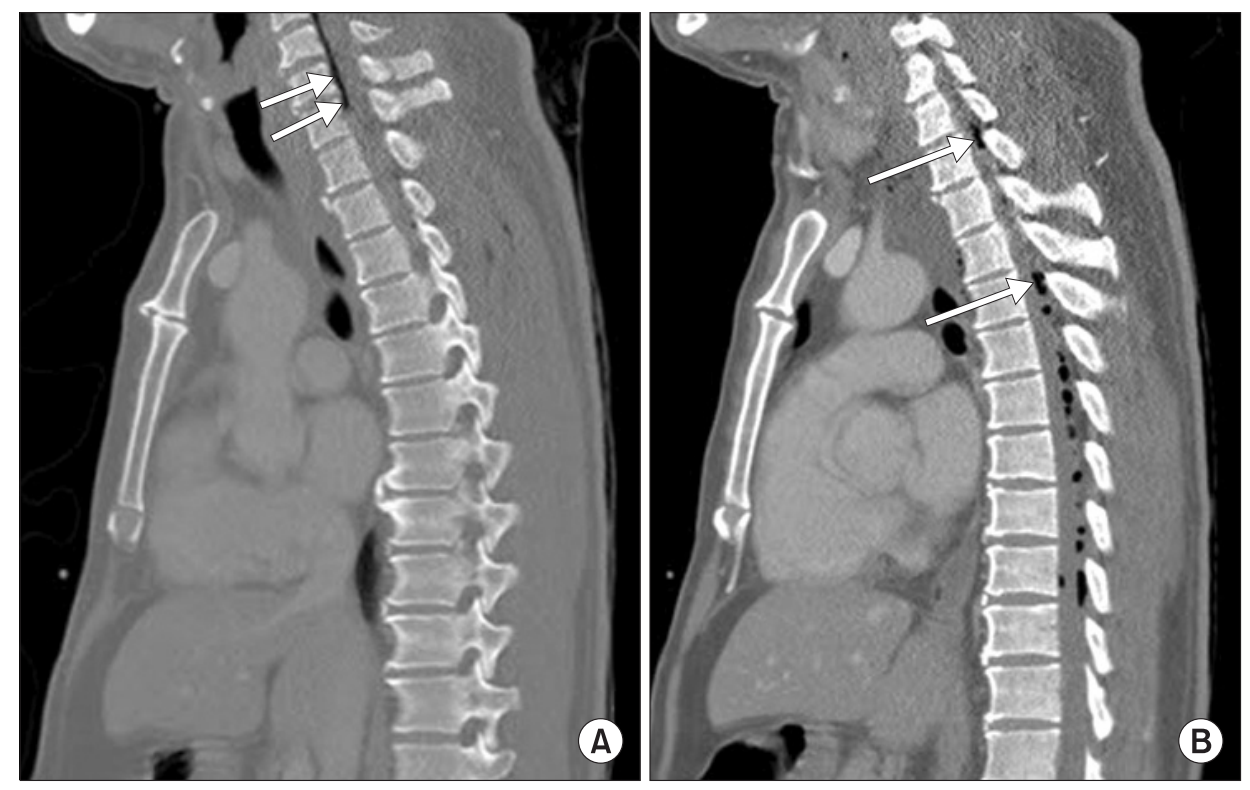

Fig. 1. Sagittal computed tomography image showing an extension of epidural air at the thoracic spine (arrows). (A) Air is present in the anterior epidural space above the T2 level. (B) Air is present in posterior epidural space below T2 level.
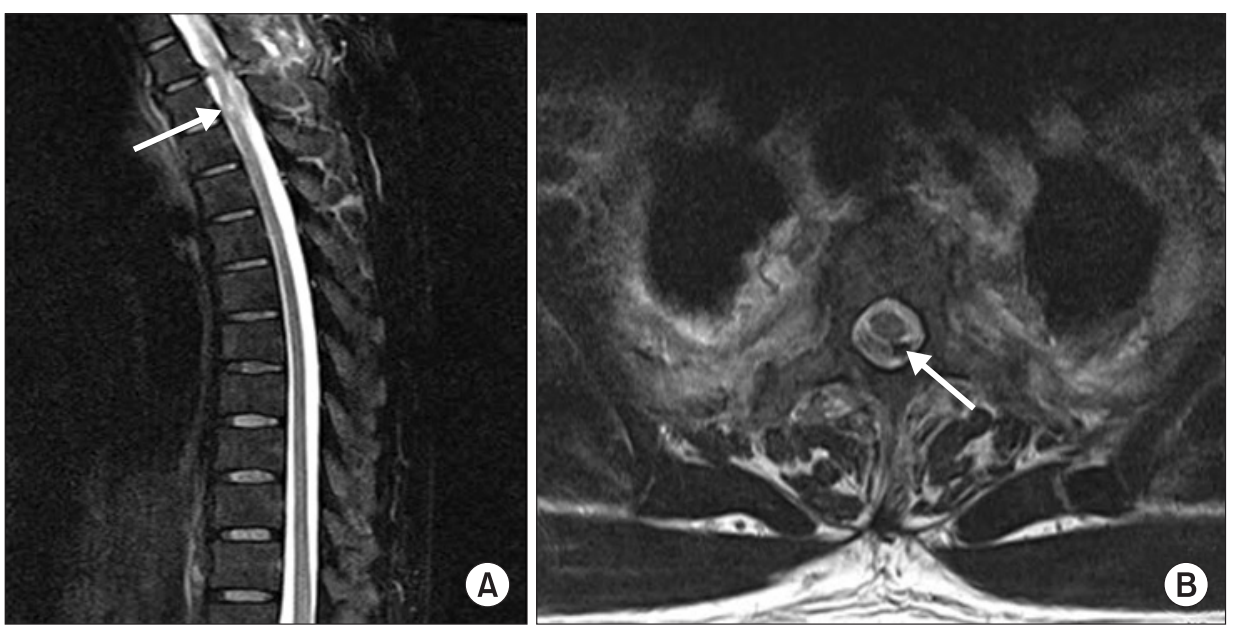

Fig. 2. Magnetic resonance imaging of thoracic spine. (A) Sagittal T2-weighted image shows a focal high signal intensity of the spinal cord at the T2 (arrow) level. (B) Axial T2-weighted image shows the posterior epidural air at the T2 (arrow) level.

was observed in the lower limbs, the deep tendon reflex was increased in the knee and ankle joints, and ankle clonus was exhibited on both ankle. Through these observations, an upper motor neuron lesion was suspected.

According to the manual muscle test, the muscular strength of both upper extremities was normal, and there was no difference in muscular strength between the lower limbs. The flexor/extensor muscles of the hip decreased to $4 / 5$, and the flexor/extensor muscles of the knee joints and the flexor muscles of the instep/sole decreased to $3 / 5$. These explain why the patient felt awkward while standing up and while walking. Consequently, the distance the patient was able to walk significantly decreased. According to the neurologic examinations, he showed hypoesthesia and muscle weakness from the dermatomere of T3, but anal constrictions could be performed voluntarily. Based on the motor and sensory nerve function, T2 in paresis was neurologically damaged. This is classified by the American Spinal Injury Association Impairment Scale as grade D, motor incomplete. From the thoracic magnetic resonance imaging (MRI) findings, the volume of air was reduced but the focal high intensity was observed in the T2 area (Fig. 2).

Electrodiagnosis was performed 4 weeks after the injury. The conduction test of the motor and sensory nerves showed a normal latency and amplitude in both lower limbs, and the latency bulbocavernosus and H-reflex in both sides were normal (Table 1). Measurements of the 
Table 1. The results of nerve conduction studies

\begin{tabular}{|c|c|c|c|c|c|c|}
\hline \multirow{2}{*}{ Nerve } & \multicolumn{3}{|c|}{ Right } & \multicolumn{3}{|c|}{ Left } \\
\hline & OL (ms) & Amplitude $^{\text {a) }}$ & $\mathrm{CV}(\mathrm{m} / \mathrm{s})$ & OL (ms) & Amplitude ${ }^{a)}$ & $\mathrm{CV}(\mathrm{m} / \mathrm{s})$ \\
\hline \multicolumn{7}{|l|}{ SNAP } \\
\hline Sural & 2.1 & 28.4 & - & 2.3 & 26.0 & - \\
\hline Superficial peroneal & 2.8 & 25.4 & - & 2.2 & 20.7 & - \\
\hline \multicolumn{7}{|l|}{ CMAP } \\
\hline Peroneal (EDB) & 4.7 & 7.8 & 48 & 4.1 & 7.5 & 47 \\
\hline Tibial (AH) & 4.5 & 8.7 & 46 & 4.6 & 7.6 & 47 \\
\hline \multicolumn{7}{|l|}{ H-reflex } \\
\hline Tibial (GCM) & 28.60 & - & - & 29.09 & - & - \\
\hline \multicolumn{7}{|l|}{ BC-reflex } \\
\hline Dorsal (BC) & 41.65 & - & - & 41.98 & - & - \\
\hline
\end{tabular}

$\mathrm{OL}$, onset latency; $\mathrm{CV}$, conduction velocity; SNAP, sensory nerve action potential; CMAP, compound muscle action potentials; EDB, extensor digitorum brevis muscle; AH, abductor hallucis muscle; GCM, gastrocnemius muscle; $\mathrm{BC}$, bulbocavernosus muscle.

${ }^{a}$ Amplitudes are measured in millivolt ( $\mathrm{mV}$, motor) and in microvolt $(\mu \mathrm{V}$, sensory).

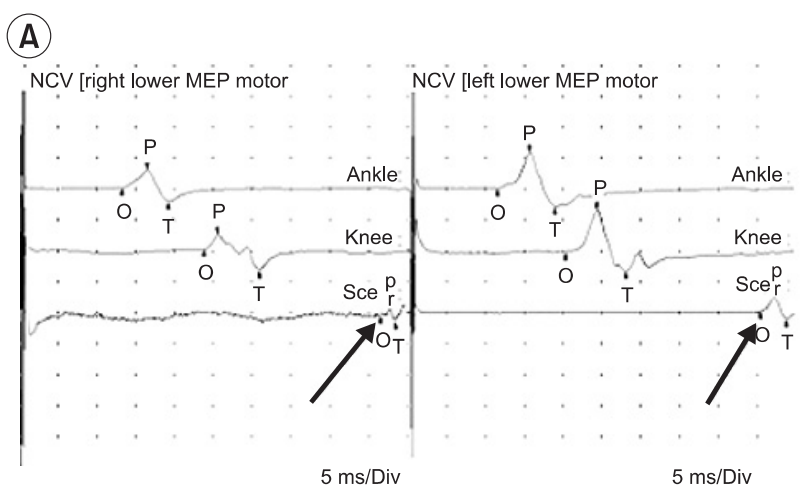

motor evoked potential at both abductor hallucis muscles were done by stimulating the head (Fig. 3A), and the somatosensory evoked potential for both tibial nerves (Fig. 3B) revealed a delay in the transcranial area. In the needle electromyographic test, a high level of abnormal spontaneous activity was observed in the paravertebral muscles and lower limb muscles from the T3 to S1 segments, which reveals the complications of incomplete thoracic myelopathy and thoracolumbar radiculopathy (Table 2).

In the hospital, the patient was enrolled in a rehabilitation program for exercising to strengthen the muscles of the lower limbs, stimulating the functional nerve roots, training for standing up, and receiving gait training. During this period, he complained of the residual urine and dysuria. Therefore, behavioral management, clean inter-

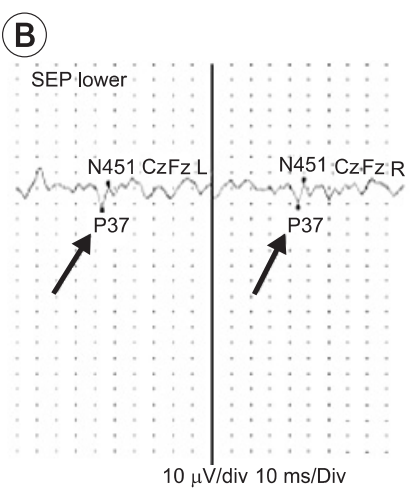

Fig. 3. Motor evoked potential (MEP) and somatosensory evoked potential (SEP) findings. (A) Onset latency of the MEP recording of the abductor halluces (Rt. 47.3 ms, Lt. $46.7 \mathrm{~ms}$ ). (B) Onset latency of tibial SEP (P37; Rt. $46.9 \mathrm{~ms}$, Lt. $46.0 \mathrm{~ms})$. NCV, nerve conduction velocity. mittent catheterization, and chemotherapy were applied for easing the urination. Two months after the injury, the manual muscle test on the hip joint flexor, knee joint extensor, and instep flexor muscles revealed the score of $5 / 5$, whereas the hip joint extensor, knee joint flexor, and sole flexor muscles revealed a score of $4 / 5$. The patient could walk long distances with ease, including the standing up. After discharge, he returned to his normal daily life and he is under continual observation.

\section{DISCUSSION}

PR is a condition characterized by air in the intraspinal canal, which is usually diagnosed incidentally while examining other coincidental injuries and disease. The air could be detected by images, such as plain radiograph, 
Table 2. The results of needle electromyography

\begin{tabular}{|c|c|c|c|c|}
\hline \multirow{2}{*}{ Muscle } & \multicolumn{2}{|c|}{ Abnormal spontaneous activity } & \multirow{2}{*}{ MUAP } & \multirow{2}{*}{ Recruitment pattern } \\
\hline & Fibrillation & PSW & & \\
\hline \multicolumn{5}{|l|}{ Right } \\
\hline Tibialis anterior & $1+$ & $1+$ & Long, poly & Complete \\
\hline Peroneus longus & $1+$ & $1+$ & Long, poly & Complete \\
\hline EHL & $1+$ & $1+$ & Long, poly & Complete \\
\hline Gastrocnemius & $2+$ & $2+$ & Long, poly & Complete \\
\hline Vastus medialis & $1+$ & $1+$ & Long, poly & Complete \\
\hline Gluteus medius & $1+$ & $1+$ & Long, poly & Complete \\
\hline Gluteus maximus & $1+$ & $1+$ & Long, poly & Complete \\
\hline \multicolumn{5}{|l|}{ Left } \\
\hline Tibialis anterior & $1+$ & $1+$ & Long, poly & Complete \\
\hline Peroneus longus & $1+$ & $1+$ & Long, poly & Complete \\
\hline EHL & $2+$ & $2+$ & Long, poly & Complete \\
\hline Gastrocnemius & $1+$ & $1+$ & Long, poly & Complete \\
\hline Vastus medialis & $1+$ & $1+$ & Long, poly & Complete \\
\hline Gluteus medius & $2+$ & $2+$ & Long, poly & Complete \\
\hline Gluteus maximus & $2+$ & $2+$ & Long, poly & Complete \\
\hline \multicolumn{5}{|l|}{ Both } \\
\hline Thoracic paraspinalis & $2+$ & $2+$ & - & - \\
\hline Lumbar paraspinalis & $2+$ & $2+$ & - & - \\
\hline
\end{tabular}

PSW, positive sharp wave; MUAP, motor unit action potential; EHL, extensor hallucis longus; Long, long duration; poly, polyphasic motor unit action potentials.

MRI, and CT scan which is especially helpful [2].

The causes of PR are generally classified into iatrogenic, non-traumatic and traumatic $[1,2]$. This iatrogenic PR can result from various surgical interventions, anesthetics, and certain processes of diagnostic techniques [3,4]. The non-traumatic etiologies include vertebral degeneration, malignancy and its associated therapy, infectious diseases by gas-forming organisms, etc. [5,6]. The PR secondary to traumatic causes is rare and it includes head, cervical, thoracic, abdominal and pelvic injuries or combinations of different injuries including spinal trauma [7].

PR can be classified as intradural or extradural, based on the locations of the air. The extradural PR is generally harmless, whereas the intradural PR is frequently associated with major trauma, and it is used as a marker for severe injuries [1].

The pathway through which the air spreads from the posterior mediastinum to the epidural space is unclear. One possible pathomechanism for epidural PR is that the air present in the posterior mediastinum cuts through the fascial planes through the neural foramina and into the epidural space, with the driving pressure from a pneumothorax or pneumomediastinum, because there is no true envelope protecting the epidural space [8]. Another probable mechanism might be the posterior mediastinal air spreading into the epidural space by direct migration through air embolization in the mediastinal veins and vertebral vein plexus of the inner vertebral body [9]. The authors support these hypotheses that the above two mechanisms were involved compositively with the epidural PR in this case, because multiple rib fractures and hemopneumothorax occurred after the chest injury. On the other hand, it was reported that the trauma of a degenerated spinal disc, containing gas due to vacuum phenomenon, can lead to air formation in the epidural space via degenerative fibrocartilage and disc fissures [2]. This hypothesis, however, can be excluded in the present case because vacuum phenomenon was not present, and the vertebra and discs showed normal radiologic findings. 
Although only a few cases of PR have been reported in Korea, most of their causes were iatrogenic and non-trauma. One case was accompanied by myelopathy and cauda equina syndrome, and the PR occurred as a result of these degenerative diseases. In this case of PR, which occurred secondarily to trauma, only the minor symptoms relating to PR were reported without any signs of neural damage [10]. The present case is a very rare incident that occurred secondarily to traumatic epidural PR, which is accompanied by paraparesis and a decreased sensation of the lower limbs. The spinal cord injuries are believed to have resulted from the pressure and distortion in the opposite directions by the air located in anterior (above T2) and posterior (below T2) epidural space (Fig. 1).

PR is usually asymptomatic and clinically non-specific, but there are a few reported cases with patients showing discomforts and sensory disturbances. The air within the spinal cord does not tend to migrate, and they usually get spontaneously reabsorbed into the bloodstream over a period of several days. Therefore, the patients with PR were normally managed conservatively [2]. Accordingly, this patient was managed by rehabilitation programs and medications. The muscle power of his lower limbs eventually recovered sufficiently enough to allow him to walk independently. The failure of an early diagnosis and treatment can delay the recovery and worsen the prognosis.

In conclusion, the early detection, a proper neurological examination, and a rehabilitation evaluation are crucial for the PR secondary to trauma accompanied by neurological symptoms. These are expected to maximize the functional recovery of the patients for their rapid return to daily life. In addition, this condition should draw more attention in the field of rehabilitation medicine.

\section{CONFLICT OF INTEREST}

No potential conflict of interest relevant to this article was reported.

\section{REFERENCES}

1. Goh BK, Yeo AW. Traumatic pneumorrhachis. J Trauma 2005;58:875-9.

2. Oertel MF, Korinth MC, Reinges MH, Krings T, Terbeck S, Gilsbach JM. Pathogenesis, diagnosis and management of pneumorrhachis. Eur Spine J 2006;15 Suppl 5:636-43.

3. Uemura K, Behr R, Roosen K. Symptomatic intraspinal air entrapment. Br J Neurosurg 2000;14:154-6.

4. Krishnam, Mallick A. Air in the epidural space leading to a neurological deficit. Anaesthesia 2003;58:292-3.

5. Ristagno RL, Hiratzka LF, Rost RC Jr. An unusual case of pneumorrhachis following resection of lung carcinoma. Chest 2002;121:1712-4.

6. Kirzner H, Oh YK, Lee SH. Intraspinal air: a CT finding of epidural abscess. AJR Am J Roentgenol 1988;151:1217-8.

7. Newbold RG, Wiener MD, Vogler JB 3rd, Martinez S. Traumatic pneumorrhachis. AJR Am J Roentgenol 1987;148:615-6.

8. Burn JM, Guyer PB, Langdon L. The spread of solutions injected into the epidural space: a study using epidurograms in patients with the lumbosciatic syndrome. Br J Anaesth 1973;45:338-45.

9. Khodadadyan C, Hoffmann R, Neumann K, Sudkamp NP. Unrecognized pneumothorax as a cause of intraspinal air. Spine (Phila Pa 1976) 1995;20:838-40.

10. Paik NC, Lim CS, Jang HS. Cauda equina syndrome caused by epidural pneumorrhachis: treatment with percutaneous computed tomography-guided translaminar trephination. Spine (Phila Pa 1976) 2013;38:E440-3. 